\title{
A IMPORTÂNCIA DA APLICAÇÃO DA PSICOLOGIA POSITIVA NA SAÚDE E SEGURANÇA OCUPACIONAL: UMA REVISÃO ${ }^{1}$
}

\author{
THE IMPORTANCE OF APPLYING POSITIVE PSYCHOLOGY IN \\ OCCUPATIONAL HEALTH AND SAFETY: A REVIEW
}

\author{
Bárbara Machado Zimmermann² e Liana Bohrer Berni ${ }^{3}$
}

\section{RESUMO}

A Psicologia Positiva propõe o entendimento dos fatores emocionais que proporcionam um estado psicológico sadio e o fortalecimento de competências individuais e coletivas, a fim de promover melhores condições de saúde e de relacionamentos. Desta forma, a inserção da Psicologia Positiva no mercado de trabalho é fundamental para compreender e focar esforços na prevenção do sofrimento psíquico dos colaboradores devido às demandas de estresses, ansiedades, insatisfações e demais desordens emocionais. Assim, o objetivo deste artigo revisional é apresentar a importância da aplicação da Psicologia Positiva no ambiente de trabalho para a prevenção de acidentes e doenças ocupacionais, a partir da abordagem do reflexo das emoções positivas na saúde mental e no trabalho. A pesquisa foi realizada nas bases de dados eletrônicas SciELO, BVS-Psi, Periódicos CAPES e Portal de Periódicos de Ciências Humanas e Filosofia utilizando os descritores "psicologia positiva", "segurança ocupacional", "saúde ocupacional", "psicologia no trabalho". Uma forma de aplicar esta teoria é utilizar instrumentos de avaliação de constructos positivos e realizar palestras com psicólogos para relacionar as emoções negativas com a ocorrência de acidentes de trabalho. Logo, para evitar o adoecimento e o sofrimento, o setor de Saúde e Segurança Ocupacional de uma organização deve estar engajado no reconhecimento e no desenvolvimento das potencialidades dos colaboradores, com o intuito de estimulá-los a trabalharem felizes e realizados, minimizando os riscos ocasionados pelas condições de trabalho, e contribuir para a excelência produtiva.

Palavras-chave: Emoções positivas, Saúde do trabalhador, Segurança no trabalho.

\section{ABSTRACT}

Positive Psychology proposes an understanding of emotional factors that provide a healthy psychological state and the strengthening of individual and collective skills in order to promote better health and relationships. Thus, the insertion of Positive Psychology in the job market is fundamental to understand and focus efforts on the prevention of the psychological suffering of employees due to the demands of stresses, anxieties, dissatisfactions and other emotional disorders. Thus, the purpose of this review article is to present the importance of applying Positive Psychology in the workplace for the prevention of accidents and occupational diseases, from the approach of the reflection of positive emotions in mental health and at work. The research was carried out in the electronic databases of SciELO, BVS-Psi, CAPES Journals and Portal of Journals on Human Sciences and Philosophy using the descriptors "positive psychology", "occupational safety", "occupational health", "psychology at work". One way to apply this theory is to use instruments to evaluate positive constructs and give lectures with psychologists to relate negative emotions with the occurrence of work accidents. So, to avoid

\footnotetext{
${ }^{1}$ Trabalho de Conclusão de Curso.

${ }^{2}$ Acadêmica do Curso de Especialização em Engenharia de Segurança do Trabalho - Universidade Franciscana - UFN.

E-mail: barbaramzesa@gmail.com

${ }^{3}$ Orientadora - Universidade Franciscana - UFN. E-mail: liana.berni@ufn.edu.br
} 
illness and suffering, the health and safety occupational sector of an organization must be engaged in the recognition and development of the potential of employees in order to encourage them to work happily and in a fulfilled manner, minimizing the risks caused by working conditions, and to contribute to productive excellence.

Keywords: Positive emotions, Employee health, Workplace safety.

\section{INTRODUÇÃO}

A busca pela compreensão do ser humano e das suas relações, e a consequente construção de práticas psicológicas e sociais adequadas e éticas faz com que seja desenvolvida uma nova visão da vida no âmbito social e cultural do indivíduo e da sociedade (MARUJO, NETO e BALANCHO, 2013). Assim, a Psicologia Positiva propõe o entendimento dos fatores emocionais que proporcionam um estado psicológico sadio e o fortalecimento de competências tanto individuais quanto coletivas (DELL'AGLIO, KOLLER e YUNES, 2006).

Esta nova perspectiva, fundada por Martin Seligman, está dividida em três níveis de atuação: (1) o nível básico ou subjetivo, que diz respeito ao estudo das emoções positivas - a felicidade, o bem-estar, a satisfação, a esperança, o otimismo e outros construtos; (2) o nível individual, que diz respeito aos traços e características individuais positivas, como as forças e as virtudes do indivíduo - afeto, perdão e (3) o nível grupal, que se refere as virtudes cívicas, aquelas que contribuem à felicidade coletiva, tais como o altruísmo, a responsabilidade, a tolerância e a ética no trabalho (BEDIN e ZAMARCHI, 2019; PUREZA et al., 2012).

Desta forma, a base desta teoria está fundamentada no bem-estar e na felicidade, bem como no desenvolvimento positivo das virtudes humanas, as quais estão associadas às forças de caráter (PUREZA et al., 2012). Ou seja, estes aspectos emocionais construtivos visam um crescimento pessoal, uma satisfação com a vida e o trabalho, uma tranquilidade, trazendo benefícios para a saúde física e mental do indivíduo, como a autorrealização e a melhoria no desempenho profissional e nas relações interpessoais (BEDIN e ZAMARCHI, 2019; HANSON e MENDIUS, 2017). Logo, a saúde física e mental do trabalhador impacta diretamente na saúde organizacional e nos níveis de bem-estar individual e coletivo (MARUJO, NETO e BALANCHO, 2013).

As Normas Regulamentadoras (NRs) 20 e 37 descrevem os riscos psicossociais como uma influência na saúde mental dos trabalhadores provocados pelas tensões da vida diária, pressão e deficiências na gestão do trabalho, podendo ter efeitos adversos a nível psicológico, físico e social, como o estresse, o esgotamento e a depressão. Neste contexto, o uso da Psicologia Positiva se faz necessário como meio de possibilitar ações que reduzam desgastes psicológicos e promovam uma condição de felicidade, engajamento, automotivação, qualidade de vida e bem-estar aos trabalhadores, e de antemão melhorar a efetividade organizacional (BEDIN e ZAMARCHI, 2019). Deste modo, o objetivo deste artigo revisional é apresentar a importância da aplicação da teoria da Psicologia Positiva no 
ambiente de trabalho para a prevenção de acidentes e doenças ocupacionais, a partir da abordagem do reflexo das emoções positivas na saúde psíquica e no trabalho.

\section{METODOLOGIA}

Este estudo foi realizado através de uma pesquisa bibliográfica, principalmente, por meio de periódicos. Foram consultadas as publicações em língua portuguesa disponíveis nas bases de dados eletrônicas SciELO (Scientific Electronic Library Online), BVS-Psi (Biblioteca Virtual em Saúde Psicologia), Periódicos CAPES (Coordenação de Aperfeiçoamento de Pessoal de Nível Superior) e Portal de Periódicos de Ciências Humanas e Filosofia. Os descritores utilizados foram "psicologia positiva", "segurança ocupacional", "saúde ocupacional", "psicologia no trabalho" e "felicidade no trabalho". As Normas Regulamentadoras do Ministério do Trabalho e Emprego, relativas à segurança e saúde do trabalho, também foram aproveitadas para compor esta revisão.

O período estudado foi do ano de 2003 a 2018, correspondente ao início das pesquisas acerca do conceito "psicologia positiva" até as atuais normas trabalhistas de saúde e segurança e sua interação com a Psicologia. A análise dos dados foi realizada através da leitura dos documentos, que buscou identificar os conceitos teóricos de interesse para esta pesquisa, as metodologias aplicadas e os principais resultados encontrados. Neste trabalho, os resultados foram subdivididos em: psicologia positiva; legislação e instrumentos de gestão de saúde e segurança ocupacional; os constructos positivos e a saúde e segurança ocupacional.

\section{RESULTADOS E DISCUSSÃO}

\section{PSICOLOGIA POSITIVA}

A Psicologia Positiva é a ciência que estuda as forças psicológicas e as emoções positivas, a fim de promover melhores condições emocionais, de saúde e de relacionamentos, auxiliando as pessoas a terem satisfação e verem sentido na vida (MARUJO e NETO, 2011). Deste modo, defende-se uma compreensão humanizada da realidade sociocultural, através de uma visão inovadora, focando em formas de potencializar o bem e a ética, e fortalecer competências ao invés de corrigir fraquezas, apesar de reconhecer a existência da psicopatologia. Esta nova percepção, contribui para a prosperidade dos indivíduos e comunidades, aumento da felicidade e dos níveis de bem-estar, visto que investe na democracia participativa, no diálogo e otimiza a autodeterminação a partir das emoções e valores, atendendo às especificidades dos grupos e contextos envolvidos (MARUJO e NETO, 2011; PALUDO e KOLLER, 2007).

As forças são responsáveis pelo comportamento, pelo sentimento e pelo pensamento do indivíduo, ou seja, pela criação da personalidade, fator contribuinte diante das situações desafiadoras que 
surgem na vida (HANSON e MENDIUS, 2017). Assim, o desenvolvimento do otimismo, perdão, da esperança, resiliência, gratidão, motivação e da capacidade de amar torna as pessoas mais felizes e contagiam o seu entorno com estas sensações, tornando o ambiente próspero para a busca da realização pessoal e integrada (RIVERO, ARAÚJO e MARUJO, 2013). Existem instrumentos que permitem a identificação, a avaliação e a classificação das características positivas dos indivíduos, dos grupos e instituições, os quais auxiliam no aprimoramento das possibilidades de florescimento, ou seja, da expansão do potencial (PALUDO e KOLLER, 2007).

Bedin e Zamarchi (2019) e Pureza et al. (2012) apresentaram alguns instrumentos para avaliação de construtos da Psicologia Positiva, tais como: Work Engagement Scale (WES), Job Satisfaction Scale (JSS), Flourishing At Work Scale (FAWS), Satisfaction With Life Scale (SWLS), Escala de Bem-Estar Subjetivo (EBES), Subjective Happiness Scale (SHS), Escala de Auto-estima de Rosemberg (EAR). Eles constataram que esta metodologia pode ajudar indivíduos e grupos a prosperarem e desenvolverem seus pontos fortes, de modo a alcançar um estado positivo de saúde mental fundamentado nas emoções positivas. Por conseguinte, a aplicação de instrumentos para analisar as forças humanas e virtudes de caráter pessoal, como a satisfação no trabalho, satisfação na vida, bem-estar, autoestima, engajamento, fazem da Psicologia Positiva uma ferramenta de transformação social que impulsiona a vida e a sociedade.

Rivero, Araújo e Marujo (2013) afirmaram que Martin Seligman e Christopher Peterson desenvolveram um sistema de classificação para determinar virtudes e forças de caráter, como mostra o Quadro 1.

Quadro 1 - Virtudes e Forças de caráter da Psicologia Positiva.

\begin{tabular}{|l|l|}
\hline \multicolumn{1}{|c|}{ Virtudes } & \multicolumn{1}{c|}{ Forças de caráter } \\
\hline Sabedoria e Conhecimento & Criatividade, Curiosidade, Originalidade, Gosto pela Aprendizagem e Empatia \\
\hline Coragem & Autenticidade, Ousadia, Persistência e Entusiasmo \\
\hline Bondade & Amor e Inteligência Social \\
\hline Justiça & Equidade, Liderança e Trabalho em equipe \\
\hline Temperança & Autocontrole, Perdão, Modéstia e Prudência \\
\hline Transcendência & Apreciar a beleza e excelência, Gratidão, Esperança, Humor e Espiritualidade \\
\hline \multicolumn{2}{|c|}{ Fonte: Adaptado de Rivero, Araújo e Marujo (2013). } \\
\hline
\end{tabular}

Com o conhecimento e a exploração destas forças e virtudes pessoais, as quais atuam como agentes protetores e preventivos das doenças mentais, é possível obter a promoção dos direitos humanos e a melhoria da qualidade de vida individual e coletiva. Logo, desperta-se uma percepção da necessidade de uma maior consciência e intervenção sobre a condição psicossocial, os valores e as crenças, desenvolvendo e aprimorando os aspectos emocionais, cognitivos, relacionais e cívicos (MARUJO e NETO, 2011; PALUDO e KOLLER, 2007). A virtude, a atenção plena e a sabedoria são as bases do bem-estar e do desenvolvimento pessoal e estimulam, respectivamente: o controle das 
ações e dos pensamentos para gerar o bem a si mesmo e aos outros; o aprendizado do novo e, o bom senso para selecionar o que faz bem, distinguindo o que causa e extingue o sofrimento (HANSON e MENDIUS, 2017).

Assim, além de conhecer as fraquezas pessoais, é necessário fortalecer as habilidades e as capacidades dos seres humanos, pois elas auxiliarão na superação de vulnerabilidades e riscos, e, na compreensão dos potenciais humanos e das experiências positivas. Esclarecer de que forma estes fatores podem contribuir para a saúde física e o florescimento grupal, interiorizar as emoções positivas e promover a motivação e a segurança, também são ações fundamentais neste processo (HANSON e MENDIUS, 2017; PALUDO e KOLLER, 2007). Deste modo, a Psicologia Positiva estuda as forças psicológicas e das emoções positivas, individuais e coletivas, e deve ser empregada em distintos contextos como um dispositivo de mudança social.

\section{LEGISLAÇÃO E INSTRUMENTOS DE GESTÃO DE SAÚDE E SEGURANÇA OCUPACIONAL}

A saúde ocupacional se destacou no âmbito nacional, em 1943, quando entrou em vigor a Consolidação das Leis do Trabalho - CLT, marco da proteção dos direitos trabalhistas. Entretanto, foi com a publicação da Portaria 3.214/78 do Ministério do Trabalho e Emprego, que as Normas Regulamentadoras (NRs) relativas à Segurança e Medicina do Trabalho foram aprovadas, fornecendo orientações obrigatórias a serem cumpridas por empregadores para tornar o ambiente de trabalho mais saudável e seguro (AQUINO, 2014).

Dentre estas normas, a NR 4 prevê que toda empresa e órgão da administração pública que possuam empregados regidos pela Consolidação das Leis do Trabalho - CLT, deverão manter, obrigatoriamente, Serviços Especializados em Engenharia de Segurança e em Medicina do Trabalho (SESMT). A constituição deste serviço tem a finalidade de promover a saúde e proteger a integridade do trabalhador no local de trabalho e seu dimensionamento é realizado em função do grau de risco da atividade e do número total de empregados. Assim, a responsabilidade pela preservação da saúde e a garantia da segurança dos trabalhadores caberá ao SESMT, o qual será composto por Médico do Trabalho, Engenheiro de Segurança do Trabalho, Técnico de Segurança do Trabalho, Enfermeiro do Trabalho e Auxiliar ou Técnico em Enfermagem do Trabalho, os quais prestarão, essencialmente, atividade prevencionista.

Desta forma, a atuação nos serviços de Saúde e Segurança Ocupacional é multiprofissional e compete a todos os integrantes do SESMT, entre outras atribuições: determinar a utilização, pelo trabalhador, de Equipamentos de Proteção Individual - EPI, de acordo com o que determina a NR 6; responsabilizar-se tecnicamente pela orientação quanto ao cumprimento do disposto nas Normas Regulamentadoras aplicáveis às atividades da empresa; manter permanente relacionamento com a 
CIPA (Comissão Interna de Prevenção de Acidentes), valendo-se ao máximo de suas observações, além de apoiá-la, treiná-la e atendê-la, conforme dispõe a NR 5; promover a realização de atividades de conscientização, educação e orientação dos trabalhadores para a prevenção de acidentes do trabalho e doenças ocupacionais, tanto através de campanhas quanto de programas de duração permanente; analisar e registrar em documento(s) específico(s) todos os acidentes ocorridos na empresa, com ou sem vítima, e todos os casos de doença ocupacional, descrevendo a história e as características do acidente e/ou da doença ocupacional, os fatores ambientais, as características do agente e as condições do(s) indivíduo(s) portador(es) de doença ocupacional ou acidentado(s).

Dos membros da SESMT, o Engenheiro de Segurança do Trabalho é o profissional que tem a responsabilidade de analisar as condições de higiene e segurança, identificar os riscos ambientais e adotar medidas preventivas para mitigar ou eliminar práticas e condições inseguras que ocasionem acidentes e doenças ocupacionais. O mesmo, também tem a função de planejar, elaborar e implementar programas que visem à proteção, à promoção e à preservação da saúde e integridade física do trabalhador, além de verificar o cumprimento das Normas Regulamentadoras do Ministério do Trabalho e Emprego (RODRIGUES e JAHESCH, 2009).

Visto isto, é necessária a implementação de ações destinadas à promoção da melhoria das condições de segurança e saúde laborais que contribuam e garantam qualidade de vida no trabalho, bem-estar físico, psicológico e social do trabalhador, tornando o ambiente produtivo (MONIZ, 2016, RODRIGUES e JAHESCH, 2009). Os principais instrumentos que contribuem para a manutenção da segurança e saúde no trabalho envolvem: comprometimento da direção, envolvimento de colaboradores, comunicação interna eficiente, investimento em capacitação técnica, implementação de programas de segurança e saúde, adoção de medidas preventivas, definição de indicadores de desempenho e de parâmetros de avaliação (MONIZ, 2016; OLIVEIRA, OLIVEIRA e ALMEIDA, 2010).

Desta maneira, as práticas administrativas e soluções técnicas necessárias para uma excelente gestão de Segurança e Saúde Ocupacional depende do envolvimento e do comprometimento das partes interessadas (alta direção, gerentes, colaboradores) e do uso de ferramentas eficientes na identificação, na redução e no controle dos riscos ocupacionais. Essa participação conjunta na elaboração de políticas e no estabelecimento de uma cultura de segurança é determinante para atender às legislações, promover a satisfação dos trabalhadores e contribuir para o aumento da produtividade e diminuição do absenteísmo e do índice de acidentes de trabalho e doenças ocupacionais (MONIZ, 2016; OLIVEIRA, 2003, OLIVEIRA, OLIVEIRA e ALMEIDA, 2010). A adoção de medidas preventivas deve ser considerada como parte integrante da cultura da organização, visto que o desempenho do ser humano na execução de suas atividades está relacionado à condição de trabalho existente (OLIVEIRA, 2003).

Para tanto, é importante reconhecer que o diagnóstico de uma doença ocupacional e o acidente de trabalho podem apresentar significativas consequências psicológicas, sociais e financeiras. Trabalhadores acidentados e/ou aqueles portadores de doenças decorrente de condições insalubres, 
muitas vezes, ficam desempregados, perdem sua remuneração e o convívio social, além de sofrerem com depressão, ansiedade e outras emoções negativas, agravando sua saúde. Por isso, o trabalho conjunto do SESMT com psicólogos é fundamental para auxiliar o trabalhador na superação destas situações adversas (LAX, 2013).

\section{OS CONSTRUCTOS POSITIVOS E A SAÚDE E SEGURANÇA OCUPACIONAL}

A transformação nos cenários social, político, econômico e cultural configurada pela transição da vertente industrial (em que os trabalhadores eram vistos como força de trabalho) para a da informação e do conhecimento, redefiniu os modelos empresariais, tornando-os mais competitivos devido à valorização da criatividade, da qualidade dos bens e serviços e do aumento da produtividade. Como consequência desta nova concepção, os trabalhadores estão sentindo um esgotamento físico e mental que desencadeia estresse, infelicidade, riscos de acidentes e afastamentos por doenças ocupacionais (FARSEN et al., 2018; RUIZ e ARAUJO, 2012).

Apesar do trabalho oferecer inserção social, dignidade, remuneração e desenvolver as habilidades do indivíduo, muitas vezes isto não é suficiente para proporcionar felicidade e bem-estar, pois pode resultar no não usufruto de momentos de lazer e no adoecimento psicológico, devido a esta falta de equilíbrio emocional (HANSON e MENDIUS, 2017). Desta forma, o estudo da Psicologia Positiva, no contexto do trabalho, é fundamental para compreender e focar esforços na prevenção do sofrimento psíquico dos colaboradores devido às demandas de ansiedades, insatisfações e demais desordens emocionais (PUREZA et al., 2012). Essa nova abordagem tem o intuito de subsidiar políticas e práticas de gestão de pessoas, orientadas à saúde física e psíquica dos trabalhadores, tornando-os mais produtivos, desenvolvendo seus potenciais através do aumento das emoções positivas, autoestima e do bem-estar e valorizando o respeito e o coleguismo (FARSEN et al., 2018).

A prevenção dos acidentes e das doenças ocupacionais busca identificar, eliminar ou manter sob controle os riscos ocupacionais, oferecendo um ambiente saudável e seguro para seus colaboradores. Entretanto, há a necessidade da inclusão dos fatores psicossociais na análise e gestão dos riscos com a participação integrada dos colaboradores nos aspectos que envolvem as condições ambientais (higiene, saúde e segurança ocupacional) e a organização do trabalho. Cabe ressaltar que algumas Normas Regulamentadoras, tais como NR 20, NR 33, NR 34, NR 35 e NR 37, preveem a inclusão de fatores de riscos psicossociais nos exames médicos, através de avaliações psicológicas, para a consequente emissão do respectivo Atestado de Saúde Ocupacional (ASO).

A NR 37 cita exemplos de condições de trabalho que conduzem aos riscos psicossociais, tais como: cargas de trabalho excessivas, falta de clareza na definição das funções, gestão de mudanças organizacionais inadequadas, insegurança laboral, comunicação ineficaz, deficiência de apoio da parte de chefias e colegas, assédio psicológico, moral e sexual. A mesma norma deve garantir aos 
trabalhadores informações sobre os riscos psicossociais, e outros, existentes no local de trabalho, a identificação dos fatores de riscos e a adoção de medidas de controle que visem à promoção, à proteção, à recuperação e à prevenção de agravos à saúde.

Deste modo, investir na valorização e na motivação dos funcionários, proporcionar condições de trabalho seguras e saudáveis, além de oportunidades de desenvolvimento e fortalecimento das capacidades profissional e pessoal, tornam a relação empregado-empregador sadia. Assim, estabelecem entre si vínculos afetivos, tais como satisfação, envolvimento e comprometimento, os quais acarretam um estado psicológico positivo, de felicidade e, consequentemente, maior desempenho produtivo, aprendizado, lucratividade, redução do absenteísmo e da rotatividade. Ou seja, a construção de um ambiente físico e psicossocial saudável, no contexto organizacional, promove a felicidade dos envolvidos, pois engloba a implantação de ações de gestão que buscam o equilíbrio entre qualidade de vida no trabalho e momentos de lazer (FARSEN et al., 2018; HANSON e MENDIUS, 2017).

A partir desta interação entre o trabalhador e o ambiente laboral, propicia-se a valorização da cooperação, da solidariedade, da confiança, da comunicação e do reconhecimento, além de uma análise mais dinâmica das situações reais dos postos de trabalho (RUIZ e ARAUJO, 2012). Para tanto, a organização deve se voltar para o colaborador a fim de melhorar seu desempenho e bem-estar, as relações interpessoais e a perspectiva profissional, além de contribuir com o crescimento empresarial (BEDIN e ZAMARCHI, 2019). Uma forma de aplicar a Psicologia Positiva no trabalho é utilizar instrumentos de avaliação dos constructos positivos e também realizar reuniões e palestras com psicólogos, abordando assuntos como a desatenção e distração, pressão excessiva por resultados e a busca pelo prestígio profissional, relacionando estas questões com as emoções negativas e a ocorrência de acidentes (HANSON e MENDIUS, 2017).

Estes instrumentos, juntamente com as medidas que visam a proteção da saúde e segurança ocupacional, permitirão à organização a verificação da situação emocional e comportamental dos seus colaboradores. O trabalho deve proporcionar qualidade de vida, bem-estar e felicidade e, portanto, o conhecimento das forças e virtudes humanas possibilita a busca por atividades que sejam inerentes as características pessoais e que elevem os níveis de emoções positivas, proporcionando proteção contra doenças psíquicas (FARSEN et al., 2018; HANSON e MENDIUS, 2017). O equilíbrio do bem-estar físico, mental e social promove uma melhor qualidade de vida e engloba aspectos como a motivação, a satisfação, a forma de gestão organizacional e a condições de trabalho (FARSEN et al., 2018).

Essa geração de um estado emocional de felicidade, engajamento, automotivação, satisfação desperta um comportamento positivo nos funcionários, pois eles têm a percepção do zelo e da valorização que estão recebendo no seu ambiente de trabalho (BEDIN e ZAMARCHI, 2019). Esta mudança de paradigma mostra que a psicologia não é apenas o estudo da patologia, mas sim da força e da virtude humana, visto que é capaz de identificar, fomentar, desenvolver e ampliar qualidades, talentos e potencialidades (FERRAZ, TAVARES e ZILBERMAN, 2007). 


\section{CONSIDERAÇÕES FINAIS}

A necessidade de realizar uma mudança da psicopatologia voltada para o estudo das forças psicológicas e emoções positivas fez surgir a área da Psicologia Positiva. Essa nova abordagem sobre o comportamento humano reconhece que as virtudes e forças humanas podem contribuir para o florescimento e o funcionamento emocional e social saudável, desencadeando a construção de uma sociedade mais ética e feliz. Logo, trabalha-se com a ideia de que para evitar o adoecimento e o sofrimento decorrente do trabalho, devemos ter a percepção dos aspectos positivos que nos levam ao crescimento, utilizando mecanismos e estratégias que permitam o equilíbrio psicossocial, agindo no "eu" e refletindo no coletivo.

A cobrança, cada vez maior, do mercado de trabalho por resultados satisfatórios tem gerado um aumento no número de casos de depressão e ansiedade, afetando diretamente a qualidade de vida dos colaboradores. Desta maneira, para fortalecer as emoções positivas e, consequentemente, prevenir acidentes e doenças ocupacionais, é importante haver manifestações de opiniões sobre as atividades desempenhadas, cooperação, apresentações de sugestões e estimulação de uma abertura comunicativa. Isso, impede o conformismo e encoraja a criatividade do colaborador, além de contribuir para o estabelecimento e a manutenção de um ambiente que desperte a empatia, a generosidade, a moralidade, a eficiência, a dedicação, a iniciativa, a justiça, a responsabilidade e a transparência.

Conforme a NR 36, os aspectos psicossociais facilitam a compreensão das atribuições e responsabilidades de cada função, o que reflete na manutenção do diálogo aberto, de modo que os trabalhadores possam sanar dúvidas, conhecer os procedimentos para prestar auxílio em caso de emergência, estimular o tratamento justo e respeitoso nas relações pessoais e facilitar o trabalho em equipe. Dessa maneira, o setor de Saúde e Segurança Ocupacional de uma organização deve estar engajado no reconhecimento e no desenvolvimento das potencialidades dos colaboradores, a fim de estimulá-los a trabalharem felizes e satisfeitos, minimizando os riscos de acidentes, desordens emocionais e doenças ocupacionais ocasionadas pelas condições de trabalho, além de contribuir para a autorrealização e excelência produtiva.

Planejar e implementar mudanças de visão, valores e de gestão de pessoas e processos em uma organização são um desafio. Entretanto, o cenário atual é marcado pela constante necessidade de adaptações legais para a manutenção da saúde e segurança ocupacional, as quais ocasionam efeitos positivos sobre o fator homem e a produtividade. Diante deste contexto, a Psicologia Positiva contempla a ideia de que os objetivos organizacionais devem ir ao encontro das competências individuais de seus colaboradores para que haja bem-estar e satisfação profissional e pessoal. Portanto, a inserção dos constructos positivos nas práticas de trabalho tem potencial para a construção de um ambiente saudável e seguro, com foco na prevenção de acidentes e de doenças ocupacionais. 


\section{REFERÊNCIAS}

AQUINO, A. S. F. Saúde ocupacional. Natal: Instituto Federal de Educação, Ciência e Tecnologia. 2014. $94 \mathrm{p}$.

BEDIN, L. M.; ZAMARCHI, M. Florescimento no trabalho: Revisão integrativa da literatura. Revista Psicologia: Organizações e Trabalho, v. 19, n. 1, p. 549-554, 2019.

BRASIL. Ministério do Trabalho e Emprego. NR 4 - Serviços especializados em Engenharia de Segurança e em Medicina do Trabalho. Portaria MTPS n ${ }^{0}$ 510, 29 de abril de 2016. D.O.U. 02 mai. 2016.

BRASIL. Ministério do Trabalho e Emprego. NR 20 - Segurança e saúde no trabalho com inflamáveis e combustíveis. Portaria $\mathrm{MTb}^{\circ}{ }^{860}$, de 16 de outubro de 2018. D.O.U. 17 out. 2018.

BRASIL. Ministério do Trabalho e Emprego. NR 33 - Segurança e saúde nos trabalhos em espaços confinados. Portaria MTE nº 1.409, 29 de agosto de 2012. D.O.U. 31 ago. 2012.

BRASIL. Ministério do Trabalho e Emprego. NR 34 - Condições e meio ambiente de trabalho na indústria da construção, reparação e desmonte naval. Portaria MTb nº 836, de 09 out. 2018. D.O.U. 10 out. 2018.

BRASIL. Ministério do Trabalho e Emprego. NR 35 - Trabalho em altura. Portaria MTb nº 1.113 , de 21 de setembro de 2016. D.O.U. 22 set. 2016.

BRASIL. Ministério do Trabalho e Emprego. NR 36 - Segurança e saúde no trabalho em empresas de abate e processamento de carnes e derivados. Portaria MTb $\mathrm{n}^{\circ} 1.087$, de 18 de dezembro de 2018. D.O.U. 19 dez. 2018.

BRASIL. Ministério do Trabalho e Emprego. NR 37 - Segurança e saúde em plataformas de petróleo. Portaria MTb no 1.186, de 20 de dezembro de 2018. D.O.U. 21 dez. 2018.

DELL'AGLIO, D. D.; KOLLER, S. H.; YUNES, M. A. M. Uma Breve Reflexão Crítica sobre Psicologia Positiva e Resiliência. Revista Semestral da Associação Brasileira de Psicologia Escolar e Educacional (ABRAPEE), v. 10, n. 1, p. 133-143, 2006. 
FARSEN, T. C. et al. Qualidade de vida, Bem-estar e Felicidade no Trabalho: sinônimos ou conceitos que se diferenciam?. Interação em Psicologia, v. 22, n. 1, p. 31-41, 2018.

FERRAZ, R. B.; TAVARES, H.; ZILBERMAN, M. L. Felicidade: uma revisão. Revista de Psiquiatria Clínica, v. 34, n. 5, p. 234-242, 2007.

HANSON, R.; MENDIUS, R. O cérebro de Buda: Neurociência prática para a felicidade. Editora: Alaúde. São Paulo. 2017.

LAX, M. B. Saúde Ocupacional na região central do estado de Nova York: um ambulatório de doenças ocupacionais com financiamento público 25 anos depois. Revista Brasileira de Saúde Ocupacional, v. 38, p. 149-161, 2013.

MARUJO, H. Á.; NETO, L. M. Investigação transformativa e apreciativa em Psicologia Positiva: um elogio à subjetividade na contemporaneidade. Estudos Contemporâneos da Subjetividade, v. 1, n. 1, p. 5-21, 2011.

MARUJO, H. Á.; NETO, L. M.; BALANCHO, L. S. F. Emergência, desenvolvimento e desafios da psicologia positiva: Da experiência subjetiva à mudança social. Estudos Contemporâneos da Subjetividade, v. 3, n. 2, p. 179 - 201, 2013.

MONIZ, A. P. B. Saúde ocupacional no século XXI: qual o papel do médico de família?. Revista Portuguesa de Medicina Geral e Familiar, v. 32, p. 372-374, 2016.

OLIVEIRA, J. C. Segurança e saúde no trabalho uma questão mal compreendida. São Paulo em perspectiva, v. 17, p. 3-12, 2003.

OLIVEIRA, O. J.; OLIVEIRA, A. B; ALMEIDA, R. A. Gestão da segurança e saúde no trabalho em empresas produtoras de baterias automotivas: um estudo para identificar boas práticas. Produção, v. 20, n. 3, p. 481-490, 2010.

PALUDO, S. S.; KOLLER, S. H. Psicologia Positiva: uma nova abordagem para antigas questões. Paidéia, v. 17, n. 36, p. 9-20, 2007.

PUREZA, J. R. et al. Psicologia positiva no Brasil: uma revisão sistemática da literatura. Revista Brasileira de Terapias Cognitivas, v. 8, n.2, p.109-117, 2012. 
RIVERO, C.; ARAÚJO, M. A.; MARUJO, H. Á. Moral e Felicidade: Possibilidades para uma Sociedade Equifeliz. Estudos Contemporâneos da Subjetividade, v. 3, n. 2, p. 229-246, 2013.

RODRIGUES, M. L.; JAHESCH, Z. M. O profissional de engenharia de segurança do trabalho e a prevenção de acidentes e doenças laborais. Caderno de administração, v. 17, n. 2, p. 26-35, 2009.

RUIZ, V. S.; ARAUJO, A. L. L. Saúde e segurança e a subjetividade no trabalho: os riscos psicossociais. Revista Brasileira de Saúde Ocupacional, v.37, n. 125, p. 170-180, 2012. 